\title{
Modified Extracorporeal Reduction of the Mandibular Condylar Neck Fracture
}

\author{
Min-Keun Kim, Kwang-Jun Kwon, Seong-Gon Kim, Young-Wook Park, \\ Jwa-Young Kim ${ }^{1}$, Hae-Yong Kweon ${ }^{2}$ \\ Department of Oral and Maxillofacial Surgery, College of Dentistry, Gangneung-Wonju National University, \\ ${ }^{1}$ Department of Oral and Maxillofacial Surgery, Hallym University College of Medicine, \\ ${ }^{2}$ Sericultural and Apicultural Materials Division, National Academy of Agricultural Science, Rural Development Administration
}

\begin{abstract}
There are many treatment options in management of mandibular condylar neck fractures. Closed reduction is the most conservative treatment; however, achievement of anatomic reduction is difficult, and there are some risks of mandibular functional impairment. Open anatomic reduction and internal fixation have some advantages; therefore, many oral and maxillofacial surgeons have attempted to achieve anatomic reduction through the open approach and extracorporeal reduction and fixation. However, when using this method, there is some risk of resorption of the fractured mandibular condylar head. Therefore, we designed a modified extracorporeal reduction technique, without detaching the lateral pterygoid muscle in order to maintain the blood supply to the fractured mandibular condylar head. We believe that this minor modification may minimize the risk of resorption of the fractured mandibular condylar head. In this article, we introduce this technique in detail, and report on two cases.
\end{abstract}

Key words: Mandibular condylar neck fracture, Mandibular condylar head resorption, Lateral pterygoid muscle, Modified extracorporeal reduction

\section{Introduction}

There are many treatment options in management of mandibular condylar neck and subcondylar fractures. These include open reduction through intraoral or extraoral approaches and closed reduction. The prognosis for each treatment is very controversial; thus, clinically, both closed reduction and open reduction are used according to the various clinical conditions of the patient[1,2]. Closed reduction is the most conservative treatment, leaves no scars, and has no surgical complications[3]; however, achieve- ment of anatomic reduction is very difficult, especially if the mandibular condylar head is deviated or severely dislocated.

If anatomic reduction is not achieved properly, the risk of mandibular functional impairment will increase. Risk factors for mandibular functional impairment are pain, perceived occlusion, and absolute difference between left and right horizontal movements[4]. Recently, there have been many attempts to use an endoscope in performance of minimally invasive open reduction; however, using this technique, the approach to the fractured site is limited.

RECEIVED September 1, 2013, REVISED September 19, 2013, ACCEPTED January 15, 2014

Correspondence to Min-Keun Kim

Department of Oral and Maxillofacial Surgery, College of Dentistry, Gangneung-Wonju National University

7 Jukheon-gil, Gangneung 210-702, Korea

Tel: 82-33-640-2753, Fax: 82-33-640-3103, E-mail: omfsmk@gwnu.ac.kr

(C) This is an open access article distributed under the terms of the Creative Commons Attribution Non-Commercial License (http://creativecommons. org/licenses/ by-nc/3.0) which permits unrestricted non-commercial use, distribution, and reproduction in any medium, provided the original work is properly cited. 
Therefore, the anatomic reduction is also difficult[5].

Open anatomic reduction and internal fixation have some advantages, which include providing adequate stability, early restoration of mandibular functions, and avoidance of prolonged maxillomandibular fixation[6]. Thus, some oral and maxillofacial surgeons have attempted to achieve anatomic reduction through the open approach and extracorporeal reduction of the mandibular condylar neck fracture (Fig. 1). However, when performing the extracorporeal reduction, there is some risk of mandibular condylar head resorption. In his review of 42 cases of extracorporeal reduction, Park et al.[7] reported that man-

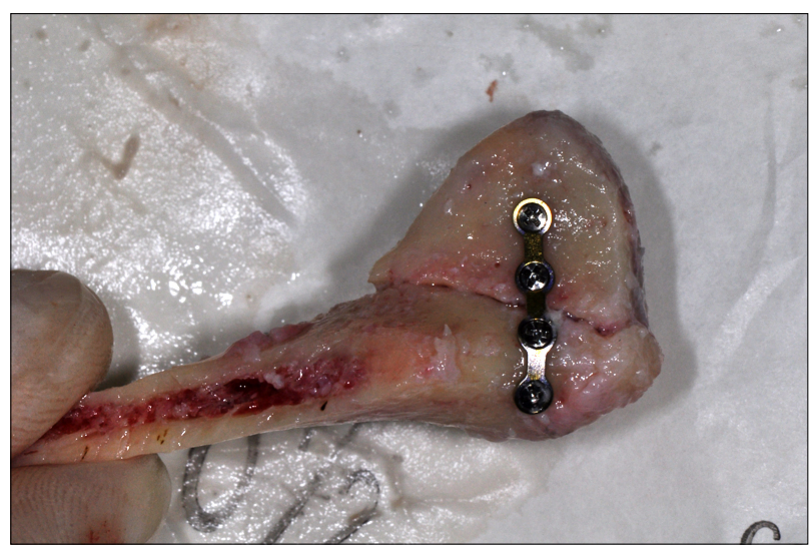

Fig. 1. Traditional extracorporeal reduction of a condylar neck fracture. dibular condylar resorption was 6.539 times more likely to occur in the extracorporeal reduction group than in those who received the closed treatment. If the mandibular condylar head is resorbed severely and causing functional impairment, the surgeon should consider reconstruction of the resorbed condylar portion through a costochondral or iliac bone graft. These risks impose heavy burdens on both the surgeon and the patient. Therefore, we designed a

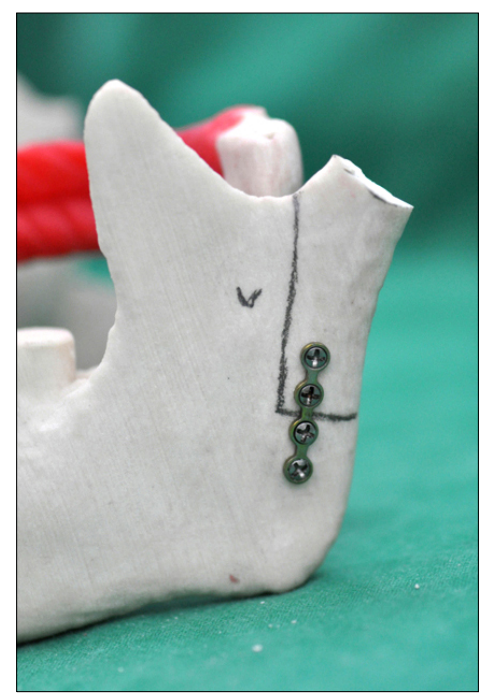

Fig. 2. Drawing of the vertical and horizontal osteotomy lines. The 4-hole miniplate was adapted before the osteotomy. The plate remembered its original position and made repositioning of osteotomized bony segment easier and more accurate later.
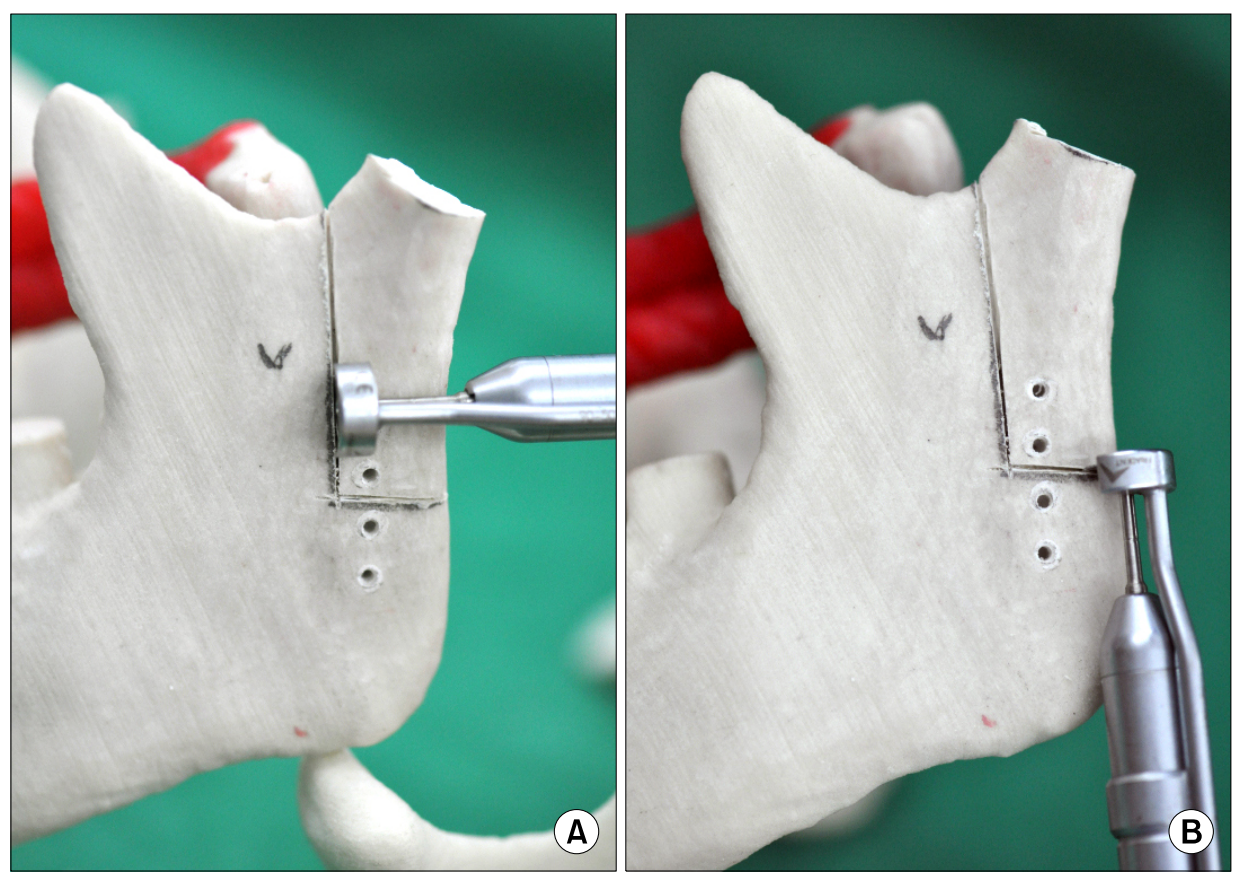

Fig. 3. (A) Sawing through the vertical osteotomy line. The surgeon should check the position of the mandibular ligula, and the osteotomy line should be positioned more posterior than the mandibular ligula. (B) Sawing through the horizontal osteotomy line. There should be steps for the vertical stop and some room inferiorly for plating. 
modified extracorporal reduction without detaching the lateral pterygoid muscle in order to maintain the blood supply to the fractured mandibular condylar head, thereby minimizing the risk of resorption of the fractured mandibular condylar head. In this article, we introduce this technique in detail, and report on two cases, each with one-year follow-up.

After using the retromandibular approach to the fractured area, we identified the sigmoid notch and marked the vertical and horizontal osteotomy lines. The vertical osteotomy line should be located more posteriorly than the mandibular lingula, thereby not causing damage to the inferior alveolar neurovascular bundles. In addition, horizontal osteotomy lines were marked, leaving some bones inferiorly in order to make a step for keeping the vertical ramal height constant. Before performance of the osteotomy, a 4-hole miniplate or one more miniplate was adapted previously for more convenient and accurate repositioning of the osteotomized segment later (Fig. 2). Vertical and horizontal osteotomies were performed using a microsaw, chisel, and mallet through the osteotomy lines, which were drawn previously (Fig. 3). When cutting the lingual cortical bone, lingual soft tissue should be protected by an instrument in order to prevent damage to the vessels and nerve bundles. The mandibular condylar head, which was not detached from the lateral pterygoid muscle, was pulled inferiorly using a wire for anatomic reduction, and fixed with two metal plates (Fig. 4, 5). Mandibular condylar head repositioning was performed (Fig. 6). The reduced proximal segment was repositioned to its original position with two 4-hole miniplates, which were adapted previously (Fig. 7). We confirmed the opening and closing movements of the mandible along with the lateral and protrusive movements. In addition, routine layer suturing was performed.

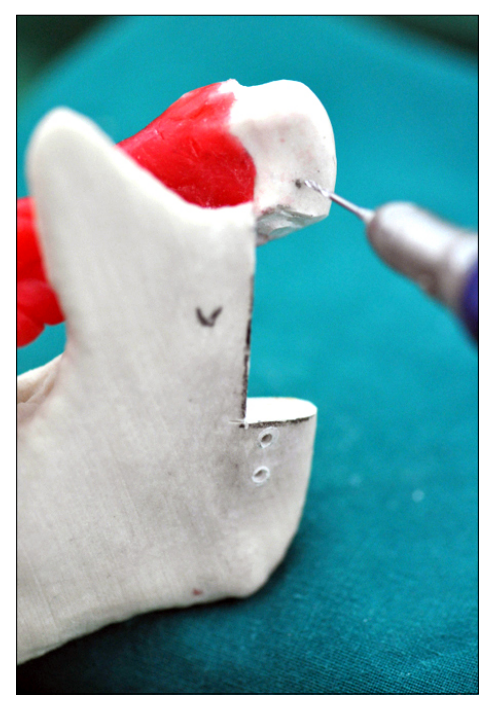

Fig. 4. Drilling was performed on the condylar neck area. We can pull the mandibular condylar head more inferiorly using wire engaged to this drilled hole.
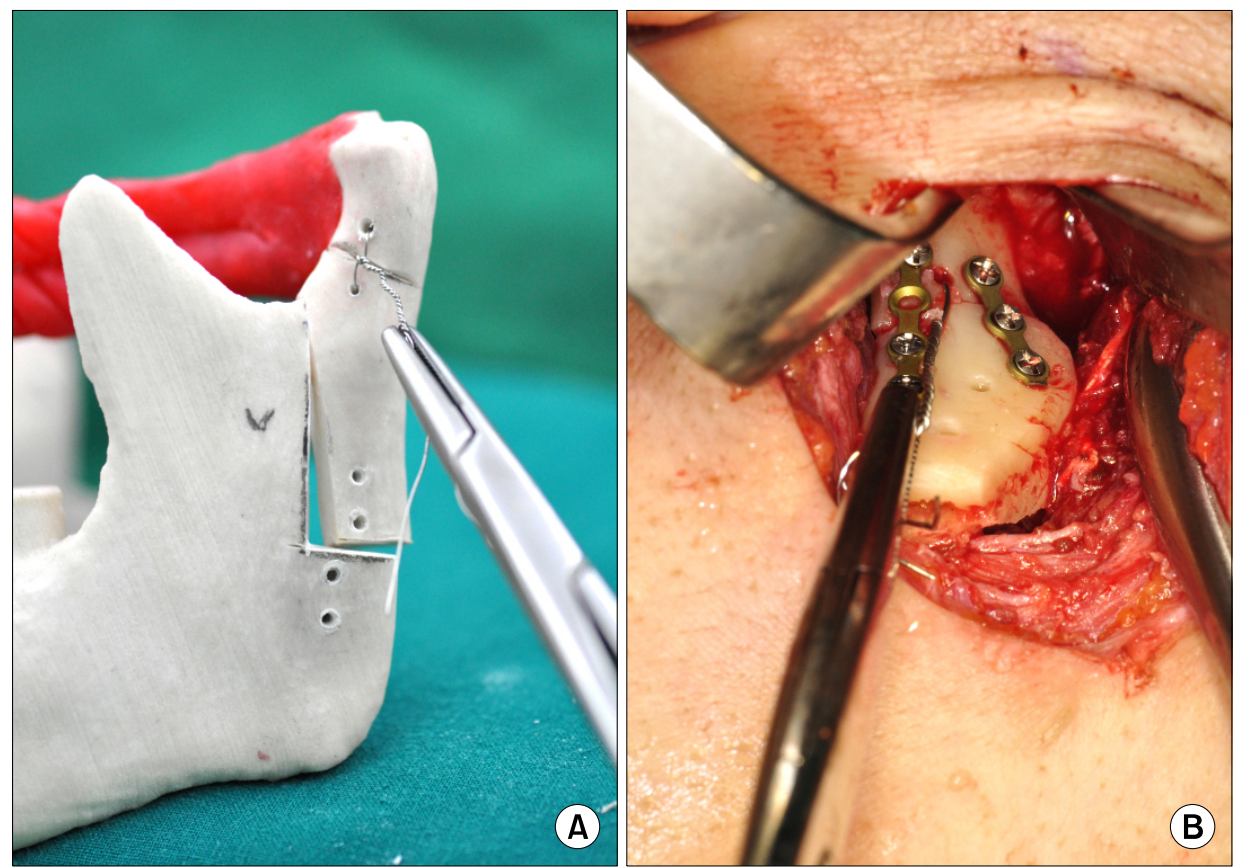

Fig. 5. (A) The mandibular condylar head and osteotomized bony segment were reduced by wire. (B) The reduced fracture site was fixed with two metal miniplates. 


\section{Case Report}

\section{Case 1}

A 25-year-old male sustained a right mandibular condylar neck fracture in a slip down accident. The mandibular condylar head was dislocated to the medio-inferior position (Fig. 8A). We reduced the fractured portion using the technique described above (Fig. 8B). Postoperative radio-

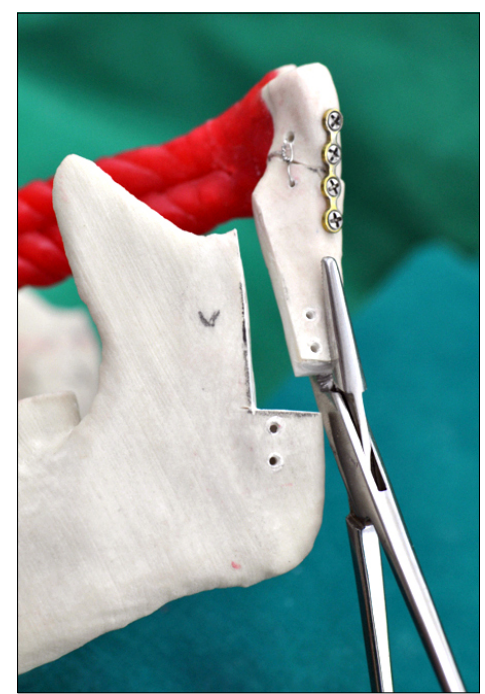

Fig. 6. The reduced condylar head was repositioned to its original position within its fossa. graphs showed a good anatomical reduction of the fracture site (Fig. 8C). Routine antibiotics and analgesics were administered postoperatively for seven days. For the first two weeks, rubber guiding and passive opening exercise were performed and a soft diet was recommended. Active mouth opening was performed postoperatively at two weeks. During the first postoperative year, there were no signs of mandibular condylar resorption (Fig. 8D). The patient exhibited stable occlusion and excellent mouth opening and lateral and protrusive mandibular movements (Fig. 8E).

\section{Case 2}

An 18-year-old male sustained a left mandibular condylar neck fracture due to blunt force trauma from his friends. The mandibular condylar fragment was displaced with lateral overlap, and there was some loss of vertical ramal height (Fig. 9A). We reduced the fractured portion using the same technique (Fig. 9B). Postoperative care was administered in the same manner. During the first postoperative year, there were no signs of mandibular condylar resorption (Fig. 9C) and the patient showed a very good functional result. During performance of the second surgery for plate removal, we were able to confirm the remodeling state of the previously osteotomized site and the fractured site (Fig. 9D).

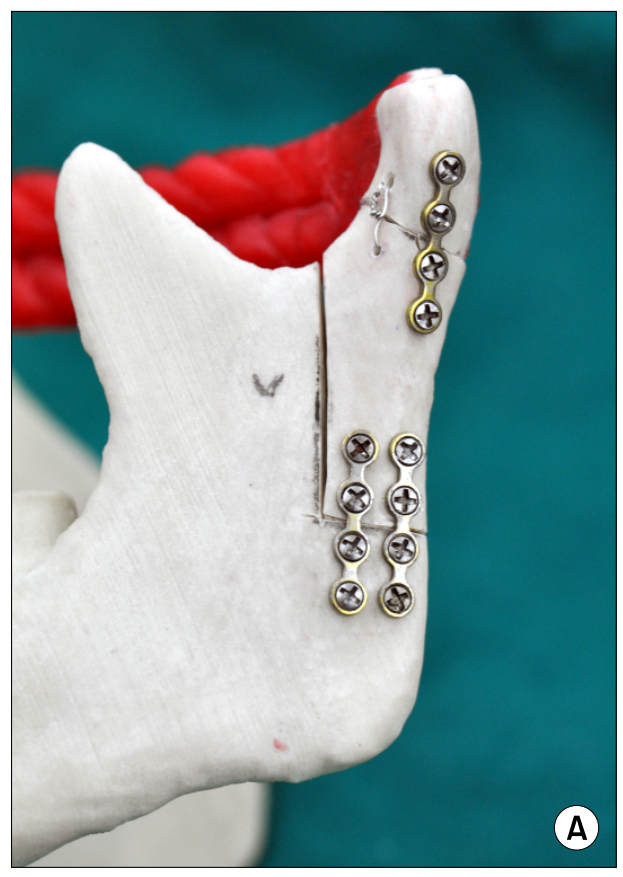

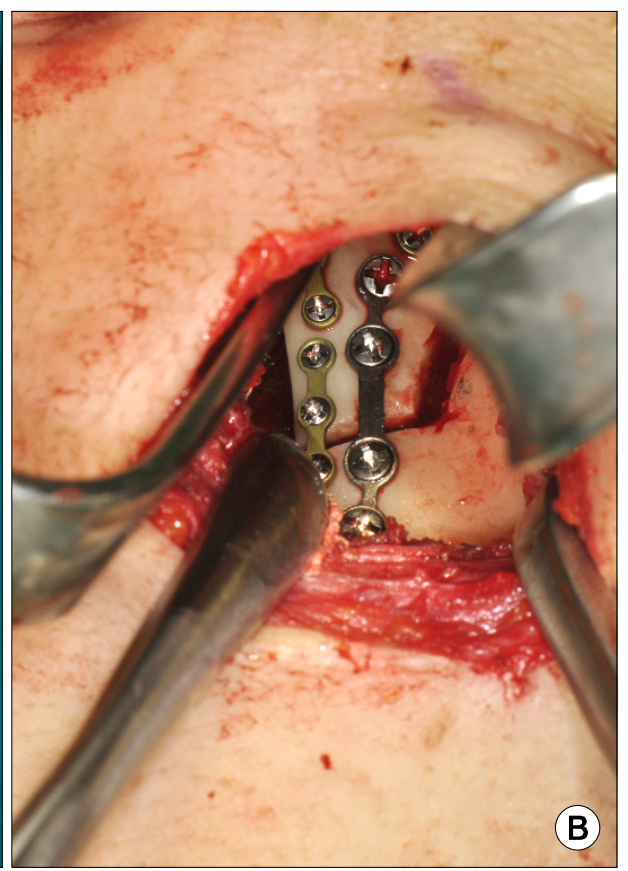

B
Fig. 7. (A) The reduced proximal segment was repositioned and fixed to its own position using two 4-hole miniplates. (B) Clinical photo of the repositioned proximal segment. 

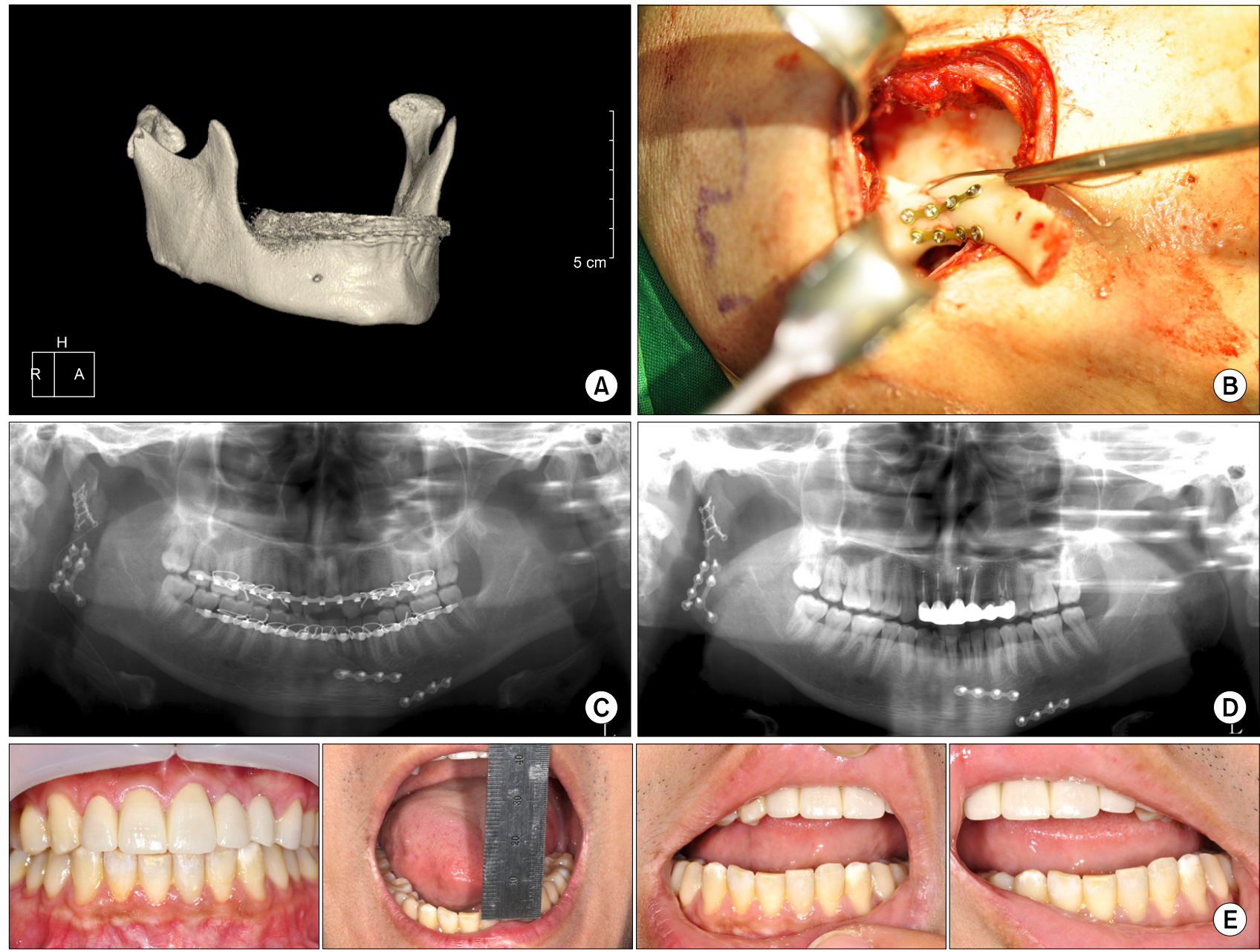

Fig. 8. (A) Preoperative computed tomography shows that the mandibular condylar head was dislocated to the medio-inferior position. (B) The clinical photo shows that we pulled the condylar head inferiorly using wire and attained anatomic reduction and fixation using two microplates. (C) Postoperative day 1 panoramic radiograph shows good anatomic reduction of the fractured mandibular neck area. (D) Postoperative 13-month panoramic radiograph shows stable bony remodeling without resorption of the condylar head. (E) The patient showed a good functional result at postoperative 13 months.

\section{Discussion}

The various treatments for a mandibular condylar neck fracture are quite controversial. Although the anatomic reduction is ideal, the surgical approach to the fractured site is very difficult and extracorporeal reduction and fixation has some risk of bony resorption of the mandibular condylar head; thus, these risks impose heavy burdens on both the surgeon and the patient. Excessive resorption of the mandibular condylar head can occur approximately $27 \%$ to $89 \%$ of the time[8,9].

This technical note introduces the retromandibular approach and modified extracorporeal reduction and fixation of the mandibular condylar neck and subcondylar fractures without detachment of the lateral pterygoid muscle. This surgical technique allows for a safer anatomic reduction of a mandibular condylar neck and subcondylar fracture. It can minimize the risk of avascular necrosis of the mandibular condylar head by maintaining the attachment of the lateral pterygoid muscle, which is responsible for the blood supply to the mandibular condylar head[10].

In the second case, direct open reduction and internal fixation could also be a good treatment plan; however, direct internal fixation requires a more extended approach to the fractured site than our method for achievement of anatomic reduction and fixation, and this may increase the risk of facial nerve damage. Therefore, we thought that in such a case the operator can decide whether or 

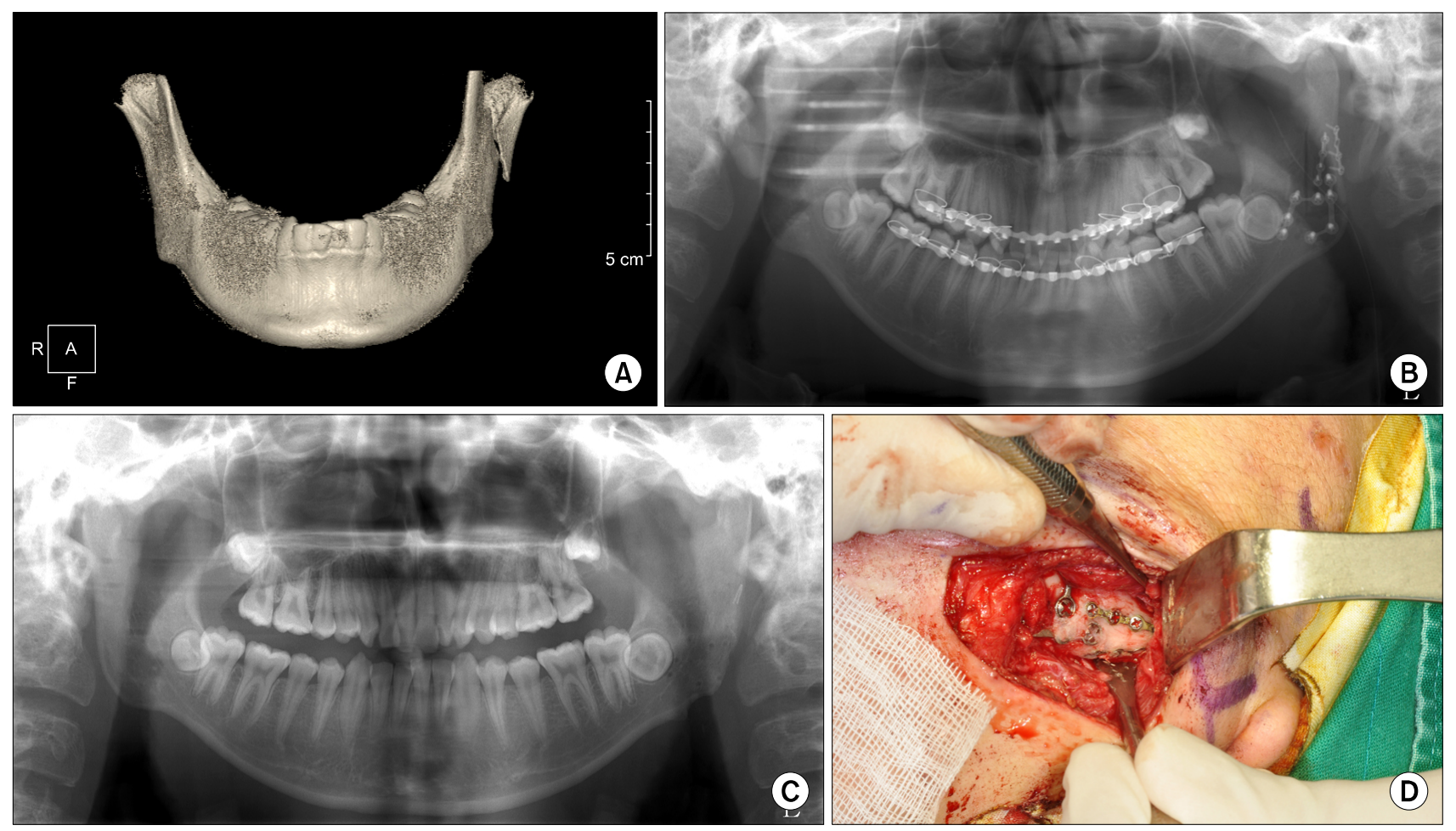

Fig. 9. (A) Preoperative computed tomography shows that the mandibular condylar fragment was displaced with lateral overlap and there was some loss of vertical ramal height. (B) Postoperative day 1 panoramic radiograph shows good anatomic reduction of the fractured mandibular neck area. (C) Postoperative 14-month panoramic radiograph shows stable bony remodeling without resorption of the condylar head. (D) The clinical photo shows remodeling of the fractured site and osteotomized sites at postoperative 14 months.

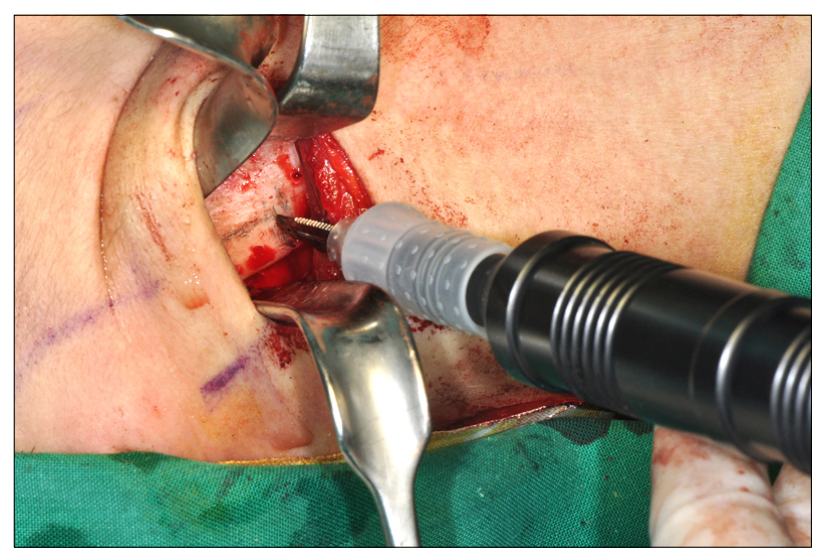

Fig. 10. Ultrasonic Osteotome hand device (BoneScalpel ${ }^{\mathrm{TM}}$; Misonix Inc.).

not additional osteotomy is necessary. When the direct internal fixation is difficult, the surgeon can consider this technique for easier performance of anatomic reduction and fixation.

In use of this technique, we have some important recommendations based on our experiences.

First, the incision line for the retromandibular approach should be designed to make an operation field that is large enough for reduction and fixation. If the fracture line is present at the high level of the mandibular condylar neck, the incision should be extended more superiorly, especially at the pterygomandibular sling and periosteal incision. Second, when planning vertical osteotomy (Fig. 3), we should check the exact position of the lingula on radiographs, such as panorama or computed tomography scan. If the lingula is positioned posteriorly, we should pay more attention in drawing an osteotomy line placed more posteriorly than the lingula. We can also use an ultrasonic osteotome hand device (BoneScalpel ${ }^{\mathrm{TM}}$; Misonix Inc., Farmingdale, NY, USA), which can reduce the risk of damage to neurovascular bundles of these areas during performance of the osteotomy (Fig. 10). Third, when performing reduction and fixation of the mandibular condylar neck, use of wire for pulling the condylar head down is very helpful (Fig. 8B). Fourth, when performing fixation of the fractured mandibular condylar neck, we can drill only after we find the most appropriate position for reduction and fixation. This is a very important point, because if the frac- 
ture level is too high, the operation field will be very limited, which will limit the amount of bony surface available for fixation, so that we usually do not have many chances for the fixation. Therefore, after we find the most appropriate position for anatomic reduction and fixation, we should perform appropriate fixation at one time.

This new method has the following advantages.

1. Functional, esthetic results can be expected from the anatomic reduction.

2. Minimal risk of excessive resorption of the mandibular condylar head since the blood supply from the lateral pterygoid muscle is maintained.

3. The severely displaced condylar fragment can be reduced anatomically.

4. Compared with the direct approach[11], this new method is more conservative and can minimize postoperative complications such as facial nerve palsy and salivary fistula.

Achievement of anatomic reduction is very difficult when using this method in a comminuted mandibular condylar neck fracture. It is the limitation of this technique. However, these types of fractures are also difficult to manage through other treatment methods. According to Park et al.[7], the type of fracture is one of the most prognostic factors of condylar fractures.

Using this technique, we experienced some postoperative swelling that was maintained for more than three months. However, the swelling subsided with passage of time. We did not perform statistical analysis of the treatment results; therefore, we will attempt to compare the resorption rate of the mandibular condylar head fragment between this technique and the conventional Nam's technique[12] in a future study.

This new technique is especially useful for growing pediatric patients whose fractured segments are severely displaced and require surgical reduction for functional and esthetic results. In this case, this method can be allowed for minimally invasive anatomic reduction. In addition, this technique can also be helpful for patients who have chronic debilitating disease and poor circulation in the mandibular condylar areas is anticipated.

\section{Acknowledgements}

This study was supported by a grant from the NextGeneration BioGreen21 Program (Center for Nutraceutical \& Pharmaceutical Materials no. PJ009013), Rural Development Administration, Republic of Korea, and was also supported by an academic research development grant from Gangneung-Wonju National University (2011-0177).

\section{References}

1. Hall MB. Condylar fractures: surgical management. J Oral Maxillofac Surg 1994;52:1189-92.

2. Walker RV. Condylar fractures: nonsurgical management. J Oral Maxillofac Surg 1994;52:1185-8.

3. Ellis E 3rd, McFadden D, Simon P, Throckmorton G. Surgical complications with open treatment of mandibular condylar process fractures. J Oral Maxillofac Surg 2000;58:950-8.

4. Niezen ET, Bos RR, de Bont LG, Stegenga B, Dijkstra PU. Complaints related to mandibular function impairment after closed treatment of fractures of the mandibular condyle. Int J Oral Maxillofac Surg 2010;39:660-5.

5. Lo J, Cheung LK. Endoscopic-assisted rigid fixation of condylar fracture: a technical note. J Oral Maxillofac Surg 2006; 64:1443-6.

6. Yang WG, Chen CT, Tsay PK, Chen YR. Functional results of unilateral mandibular condylar process fractures after open and closed treatment. J Trauma 2002;52:498-503.

7. Park JM, Jang YW, Kim SG, et al. Comparative study of the prognosis of an extracorporeal reduction and a closed treatment in mandibular condyle head and/or neck fractures. J Oral Maxillofac Surg 2010;68:2986-93.

8. Davis BR, Powell JE, Morrison AD. Free-grafting of mandibular condyle fractures: clinical outcomes in 10 consecutive patients. Int J Oral Maxillofac Surg 2005;34:871-6.

9. Iizuka T, Lindqvist C, Hallikainen D, Mikkonen P, Paukku P. Severe bone resorption and osteoarthrosis after miniplate fixation of high condylar fractures. A clinical and radiologic study of thirteen patients. Oral Surg Oral Med Oral Pathol 1991;72: 400-7.

10. Liu CK, Liu P, Meng FW, et al. The role of the lateral pterygoid muscle in the sagittal fracture of mandibular condyle (SFMC) healing process. Br J Oral Maxillofac Surg 2012;50: 356-60.

11. Croce A, Moretti A, Vitullo F, Castriotta A, Rosa de M, Citraro L. Transparotid approach for mandibular condylar neck and subcondylar fractures. Acta Otorhinolaryngol Ital 2010;30:303-9.

12. Nam IW. The condylar head and upper condylar neck fractures treated by Dr. Nam's method. J Korean Assoc Oral Maxillofac Surg 1980;6;25-30. 